\title{
Prevalência da hanseníase no período de 2013 a 2017 em Guaraí/TO
}

A hanseníase é uma doença lenta que acomete, predominantemente, adultos. Causada pelo bacilo Mycobacterium leprae, o micróbio possui alta infectabilidade, afetando nervos periféricos e pele. O Brasil assume o segundo lugar em casos registrados no mundo; cerca de $94 \%$ dos casos nas Américas estão registradas no país. Considerado sério problema de saúde pública, conhecer a epidemiologia da doença é uma ferramenta importante no combate à endemia. O Tocantins, por se tratar de um estado com alta taxa de prevalência da doença, necessita de estudos mais amplos, sobretudo nos municípios com maiores casos notificados. Verificou-se em Guaraí que há uma prevalência de hanseníase, embora o número tenha diminuído. Porém, necessita de uma constante vigilância para o combate efetivo dos enfermos e proteção das populações vulneráveis. O objetivo deste estudo foi analisar a incidência e prevalência de hanseníase no município de Guaraí/TO.

Palavras-chave: Hanseníase; Diagnóstico; Incidência; Prevalência.

\section{Prevalence of leprosy in the period from 2013 to 2017 in Guaraí/TO}

\begin{abstract}
Leprosy is a slow disease that affects, predominantly, adults. Caused by the Mycobacterium leprae bacillus, the microbe has high infectivity, affecting peripheral nerves and skin. Brazil takes second place in registered cases in the world; about $94 \%$ of the cases in the Americas are registered in the country. Considered a serious public health problem, knowing the epidemiology of the disease is an important tool in the fight against the endemic. Tocantins, because it is a state with a high prevalence rate, requires broader studies, especially in municipalities with the highest reported cases. It was found in Guarai that there is a prevalence of leprosy, although the number has decreased. However, it needs constant vigilance to effectively combat the sick and protect vulnerable populations. The objective of this study was to analyze the incidence and prevalence of leprosy in the municipality of Guaraí/TO.
\end{abstract}

Keywords: Leprosy; Diagnosis; Incidence; Prevalence.

Topic: Enfermagem em Doenças Infecciosas

Reviewed anonymously in the process of blind peer

Míriam Silva Azevedo

Faculdade Guaraí, Brasil

http://lattes.cnpq.br/5009555919327947

miryan-costa@hotmail.com

Eliane Fátima Gonçalves

Faculdade Guaraí, Brasil

http://lattes.cnpq.br/1984676943131530

elianefg1000@hotmail.com

Aluísio Vasconcelos Carvalho

Faculdade Guaraí, Brasil

http://lattes.cnpq.br/5200758055263996

http://orcid.org/0000-0002-3793-3133

aluisiovasconcelos@gmail.com
Received: $14 / 12 / 2017$

Approved: 26/12/2017
Referencing this:

AZEVEDO, M. S.; GONÇALVES, E. F.; CARVALHO, A. V.. Prevalência da hanseníase no período de 2013 a 2017 em Guaraí/TO. Scire Salutis, v.7, n.2, p.58-64, 2017. DOI: http://doi.org/10.6008/SPC22369600.2017 .001 .0007 


\section{INTRODUÇÃO}

A hanseníase é uma doença infectocontagiosa causada pelo Mycobacterium leprae, que possui afinidade por células cutâneas e nervos periféricos. Doença de evolução lenta que se manifesta, principalmente, através de um conjunto de sinais e sintomas dermatoneurológico (ARAÚJO, 2003). O comprometimento de nervos periféricos é a principal característica da doença, conferindo-lhe grande potencial para provocar incapacidades físicas que podem, inclusive, evoluir para deformidades (GOMES, 2005). No entanto, seu diagnóstico precoce e tratamento adequado evitam a evolução da doença, e consequentemente, impedem a instalação das incapacidades físicas por ela provocadas.

A proposta da Organização Mundial de Saúde (OMS) de eliminação da hanseníase é compreendida como uma redução da taxa de prevalência a menos de uma pessoa afetada por 10.000 habitantes, sendo que os países endêmicos deverão alcançar essa meta até 2020. Foi um marco importante para redefinir as atividades de controle da doença (BRASIL, 2015).

Vários fatores contribuem para a permanência do alto índice de prevalência da hanseníase no país, impedindo que sua eliminação se torne realidade; dentre eles, a permanência de casos não diagnosticados responsáveis pela manutenção da cadeia de transmissão da doença na população. A estratégia atual de controle da hanseníase no Brasil baseia-se na detecção precoce de casos e tratamento dos casos com poliquimioterapia (BRASIL, 2000). Conforme sugerido pela Organização Mundial de Saúde (OMS), o controle da hanseníase no País está descentralizado, sendo realizado pelo município, visando garantir que os serviços de diagnóstico e tratamento sejam distribuídos de forma equitativa e acessível (SANTOS et al., 2008).

No Brasil, a hanseníase é uma doença de notificação compulsória em todo território nacional e de investigação obrigatória. Assim, a cada caso diagnosticado, deve ser notificado na semana epidemiológica de ocorrência do diagnóstico, utilizando-se a ficha de notificação e investigação do Sistema de Informação de Notificação de Agravos (SINAN) em toda rede de atenção à saúde do SUS (BRASIL, 2015). Dessa forma, o presente estudo avalia, através da prevalência, se a endemia está em expansão ou caminha para a eliminação na cidade de Guaraí/TO, bem como seus aspectos epidemiológicos.

\section{METODOLOGIA}

\section{Área de estudo}

O estado do Tocantins, o mais novo da Federação, com apenas 27 anos de existência em franco crescimento, destaca-se como uma das economias mais promissoras da região norte do Brasil. De acordo com IBGE (2016), o município de Guaraí possui uma extensão territorial de $2.268 \mathrm{~km}^{2}$ e está situado na Mesorregião Ocidental do Tocantins e Microrregião de Miracema do Tocantins, distante 173km da capital do Estado (Palmas) sob as coordenadas 0850'03"S e 4830'37"O. Segundo o Instituto Brasileiro de Geografia e Estatística (IBGE), possui uma população de 23.200 habitantes, de acordo com o censo demográfico de 2010 (IBGE, 2007). 


\section{Percurso metodológico}

Trata-se de um estudo epidemiológico transversal e descritivo, que utilizou os dados dos casos registrados no caderno de hanseníase do Ministério da Saúde. Foram selecionados todos os casos novos de hanseníase notificados e confirmados em residentes de Guaraí abrangendo o período de 2013 a 2017. As variáveis do estudo foram divididas em cinco categorias de análise: incidência, sexo, faixa etária, classificação operacional, forma clínica e grau de incapacidade. $\mathrm{O}$ indicador utilizado para o estudo foi o coeficiente de incidência por 10.000 habitantes. Incluiu-se todos os casos novos registrados nesse período, e realizou análise exploratória e descritiva dos dados a partir da apuração de frequências simples absolutas e percentuais para as variáveis categóricas e organização dos resultados em tabelas e gráficos.

\section{RESULTADOS E DISCUSSÃO}

De acordo com os dados registrados no Ministério da Saúde, entre 2013 e 2017 foram registrados 90 casos novos de hanseníase na população residente no município de Guaraí/TO. O ano de 2013 foi o que teve maior número de notificações de casos novos de hanseníase, obtendo-se $43,33 \%$ do total de casos. Naquele ano, registraram-se 39 casos novos, com coeficiente de incidência de 16,8 casos/10.000 habitantes; 2014 com 4,3/10.000; 2015 com 6,3/10.000; 2016 e 2017 com 3,1/10.000 casos novos de hanseníase, conforme se nota na Quadro 01.

Quadro 01: Taxa de incidência de hanseníase em Guaraí/TO de 2013 a 2017.

\begin{tabular}{|c|c|c|c|}
\hline Ano & Número de casos novos & População residente & $\begin{array}{c}\text { Taxa de incidência/ } \\
\mathbf{1 0 . 0 0 0} \text { hab. }\end{array}$ \\
\hline 2013 & 39 & 23.200 & 16.8 \\
\hline 2014 & 10 & 24.629 & 4.0 \\
\hline 2015 & 16 & 25.149 & 6.3 \\
\hline 2016 & 17 & - & - \\
\hline 2017 & 08 & 25.642 & 3.1 \\
\hline
\end{tabular}

A hanseníase é uma infecção crônica que acomete principalmente zonas do corpo com menor temperatura, incluindo a pele e nervos. Entretanto, se for tratada nas fases iniciais da doença, o indivíduo pode obter a cura. A taxa de detecção é a base para o monitoramento da hanseníase, e determina o nível de transmissão da infecção. Além desse, outros indicadores podem ser considerados de impacto para o monitoramento dessa infecção, como a proporção de casos multibacilares e proporção de casos com grau de incapacidade entre os novos casos. A taxa de detecção de casos e o atraso no diagnóstico são dependentes de vários fatores, tais como idade, profissão, nacionalidade, endemicidade, tipo de hanseníase e método de detecção (SANTOS et al., 2008).

Outro fator arraigado aos problemas da hanseníase são a precariedade dos serviços de saúde; condições higiênicas e sanitárias, levando os pacientes à exposição do patógeno; e fatores sociais e econômicos, que evidenciam o aumento das taxas de prevalência e incidência de casos, sobretudo nas populações vulneráveis e negligenciadas (HELENE et al., 2002; ARAÚJO, 2003). 
Segundo o levantamento do Ministério da Saúde, os casos novos de hanseníase no Brasil caíram 34\% nos últimos dez anos, sendo apenas uma preocupação para as regiões centro oeste e norte do país, que ainda são consideradas regiões de endemias. O Tocantins, por se tratar de um estado novo, com 139 municípios, está em segundo lugar no rank nacional da hanseníase; Guaraí, oitavo município mais populoso do estado, uma região norte com estimativa populacional de 25.642 mil habitantes, possui 6 unidades de saúde (MONTEIRO et al., 2015).

Sabe-se que, no que se refere à incidência em Guaraí no período de 2013 a 2017, a classificação da taxa de detecção oscilou entre média de 3,1 a 16,8 casos/10.000; no ano de 2013 foi o que teve maior número de notificações de casos novos de hanseníase, com $43,33 \%$ do total de casos. Nesse ano, registraram foram registrados 39 casos novos, com coeficiente de incidência de 16,8 casos/10.000 habitantes; 2014 com 4,3/10.000; 2015 com 6,3/10.000; 2016 e 2017 com 3,1/10.000 casos novos de hanseníase. O número de casos novos predominou acentuadamente no gênero masculino no período de 2013 a 2017, como se pode observar na figura 1.

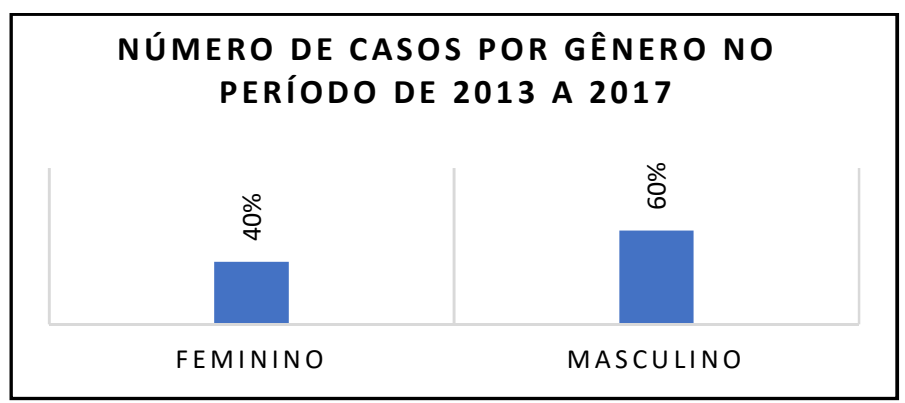

Figura 1: casos positivos de Hanseníase no município de Guaraí/TO classificados de acordo com o gênero.

Segundo a OMS, o sexo masculino teve uma maior prevalência: duas vezes mais frequentes nos casos notificados em 2001 que o sexo feminino. As relações interpessoais e de trabalho em sociedade predominantemente patriarcais levam os homens a uma maior exposição e risco de contato com indivíduo doente, favorecendo, desta forma, a propagação da doença no meio masculino (CAMPOS et al., 2005; LANA et al., 2011).

Nessa vertente, os homens têm maior contato social entre pessoas do mesmo sexo, não possuem preocupação com a saúde do corpo e a estética, o que é diferente das mulheres, que possuem programas de saúde específicos voltados para a saúde feminina, resultando em maiores oportunidades para o diagnóstico precoce da doença (MELÃO et al., 2011). Já é sabido que as mulheres desenvolvem resposta imunológica mais intensa ao $M$. Leprae que os homens, o que justifica, além de menor incidência, também a menor gravidade da patologia no sexo feminino (MIRANZI et al., 2010).

No que se refere à idade da população em estudo, a distribuição por faixa etária variou de 14 a 75 anos. Observa-se que a frequência aumentou com a idade, e a maior incidência ocorreu em indivíduos que se encontrava na faixa etária dos 40 aos 59 anos. Esta amostra condiz com a literatura, que confirma a dominação da hanseníase na faixa etária de maneira economicamente ativa (BELDA, 1976; VERONESI et al., 2002). 
Doenças que apresentam longos períodos de incubação, associadas com a demora no diagnóstico e tratamentos, são responsáveis pelo aumento no número de casos, de acordo com a progressão da idade (RODRIGUES, 2010; OLIVEIRA, 2015), o que é de grande relevância, pois esta é a fase produtiva do desenvolvimento das atividades laborais, as quais de maneira drástica podem ser interrompidas por causa do alto poder incapacitante da doença (TALHARI et al., 2014; DIAS, 2013). De acordo com os dados amostrados por meio da distribuição dos casos por faixa etária, é observada uma alta taxa de incidência em pacientes de 40 a 59 anos (41\%) e de 20 a 39 anos (32\%), ilustrado na figura 2 .

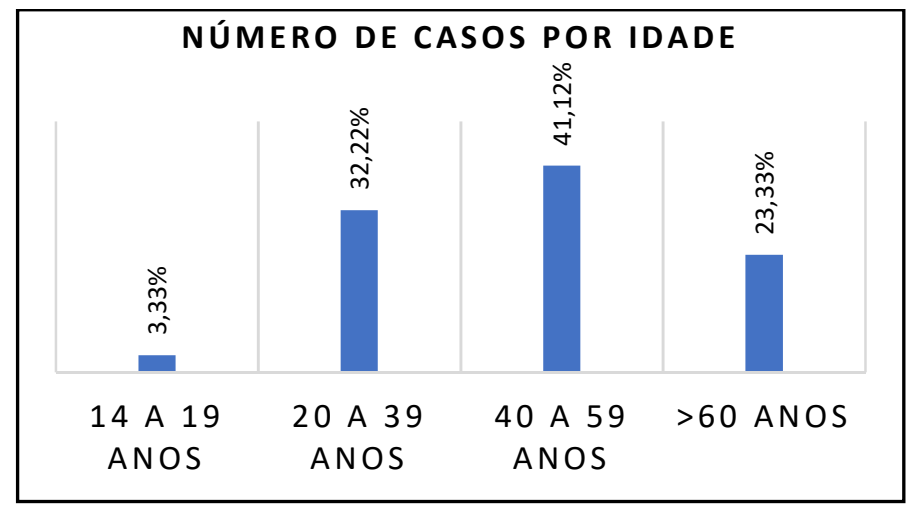

Figura 2: Número de casos de Hanseníase divididos por faixa etária.

Neste estudo, pode-se observar também o predomínio da classificação operacional dos indivíduos, que encontrou uma prevalência de $62,23 \%$ de casos multibacilares. Os resultados encontrados na presente pesquisa, em relação à classificação operacional, apresentam-se concordante com outros estudos encontrados na literatura, dentre eles Amaral (2008) e Miranzi et al. (2010).

Conforme o número de casos multibacilares aumenta, pensa-se em uma determinada estabilização temporal da endemia, quando indivíduos mais suscetíveis são afetados, poupando de forma estratégica os imunologicamente competentes, ou há uma falha no diagnóstico ocorrendo tardiamente nas populações vulneráveis que não possuem informação da doença, a precariedade ou ineficiência do sistema de atendimento primário e epidemiológico que perpetua o foco de transmissão (GOMES et al., 2005; BASSO et al., 2017; MELÃO et al. 2011).

De acordo com o Ministério da Saúde, indivíduos classificados como multibacilares representam importante fonte de infecção e manutenção da cadeia epidemiológica da endemia (BRASIL, 2015). Nas formas clínicas multibacilares, o predomínio (Dimorfa e Virchorviana) é sugestivo de diagnóstico tardio, o que leva a entender que a rede de atenção básica a saúde ainda apresenta dificuldade na detecção dos casos nas formas iniciais da doença (OLIVEIRA, 2015), o que nesta pesquisa apresentou uma prevalência de 48,89\%.

$\mathrm{Na}$ classificação operacional, a porcentagem de pacientes que apresentaram a classificação multibacilar foi de $62,23 \%$, e a classificação paucibacilar, $37,77 \%$. Na avaliação da forma clínica Dimorfa, foram maioria, com $48,89 \%$ dos casos, seguidos pela tuberculóide, com 23,33\%; vichorviana com 13,33\%; e a indeterminada, apresentando 13,33\%; e não classificados com a menor proporção dos casos, com 1,11\%, conforme se pode verificar na figura 3 . 


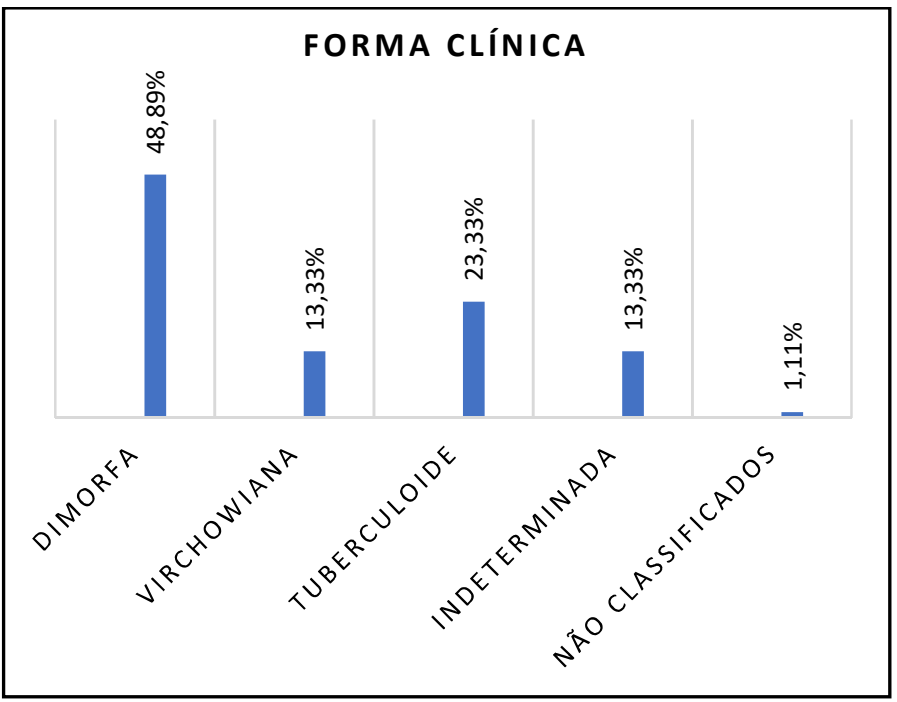

Figura 3: Forma clínica dos casos positivos de Hanseníase em Guaraí/TO.

A hanseníase dimorfa é caracterizada pela instabilidade imunológica, variando sua manifestação clínica em diversos pacientes, seja no tecido epitelial, nervos ou mesmo comprometimento sistêmico (ARAÚJO, 2003). Segundo Araújo (2003), a hanseníase virchowiana é conhecida por ter uma influência ao polo de baixa resistência manifestando nos indivíduos imunodeprimidos para o M. leprae. A forma clínica indeterminada é caracterizada pelo início da doença, já a forma tuberculóide é acometido em pacientes portadores de resistência natural induzindo imunidade celular contra o bacilo (LANA et al., 2011).

\section{CONCLUSÕES}

O Ministério da Saúde tem o compromisso de eliminação da hanseníase até 2020, ou seja, alcançar menos de 1 caso a cada 10.000 habitantes. Porém, necessita de programas efetivos de controle e prevenção principalmente nas comunidades vulneráveis. Espera-se que o presente estudo sirva como subsídio para formulação de estratégias e ações frente ao programa municipal de hanseníase, visto que os indicadores demostram há uma necessidade de redução da taxa de detecção.

Também é importante que essa detecção de novos casos ocorra em tempo oportuno e precoce, impedindo a permanência da cadeia de transmissão e que os indivíduos acometidos pela doença venham sofrer com as complicações físicas, psicológicas e até sociais provenientes destes estigmas ainda existente em nossa sociedade. Neste sentido, acredita-se que é de grande importância o aprimoramento das condições operacionais de rede de serviços e a necessidade da criação de novos projetos e linhas de combate a hanseníase no Brasil.

\section{REFERÊNCIAS}

AMARAL, E. P.; LANA, F. C. F.. Análise espacial da Hanseníase na microrregião de Almenara, MG, Brasil. Revista Brasileira de Enfermagem, v.61, p.701-707, 2008.

ARAÚJO, M. G.. Hanseníase no Brasil. Revista da Sociedade Brasileira de Medicina Tropical, v.36, n.3, p.373-382, 2003.
BASSO, M. E. M.; SILVA, R. L. F.. Perfil clínico-epidemiológico de pacientes acometidos pela hanseníase atendidos em uma unidade de referência. Revista da Sociedade Brasileira de Clínica Médica, v.15, n.1, p.27-32, 2017. 
BELDA, W.. Aspectos epidemiológicos da hanseníase no estado de São Paulo em 1974. Hansenologia Internationalis, v.1, n.1, p.11-24, 1976.

BRASIL. Portaria n.1073/GM de $\mathbf{2 6}$ de setembro de 2000. Dispõe sobre a necessidade de viabilizar a efetiva inserção das ações de controle da hanseníase na atenção básica de saúde. Brasília: DOU, 2000.

BRASIL. Ministério da Saúde. Detectar, tratar e curar: desafios e estratégias brasileiras frente à tuberculose. Boletim Epidemiológico, v.46, n.9, p.1-19, 2015.

CAMPOS, S. S. L.; RAMOS JUNIOR, A. N.; KERR-PONTES, L. R. S.; HEUKELBAC, J. H.. Epidemiologia da hanseníase no Município de Sobral, Estado do Ceará-Brasil, no Período de 1997 a 2003. Hansenologia Internationalis, v.30, n.2, p.167173, 2005.

DIAS, J. L.; GODOY, G. M. S.; AGUIAR, R. S.; GOMES, G. P. L. A.. Características determinantes entre portadores de hanseníase em uma área hiperendêmica. Revista Brasileira de Ciências da Saúde, v.11, n.38, p.32-37, 2013.

GOMES, C. C. D.; PONTES, M. A. A.; GONÇALVES, H. S.; PENNA, G. O.. Perfil clínico-epidemiológico dos pacientes diagnosticados com hanseníase em um centro de referência na região nordeste do Brasil. Anais Brasileiros de Dermatologia, v.80, n.3, p.283-288, 2005.

HELENE, L. M. F.; SALUM, M. J. L.. A reprodução social da hanseníase: um estudo do perfil de doentes com hanseníase no Município de São Paulo. Cadernos de Saúde Pública, v.18, n.1, p.101-113, 2002.

LANA, F. C. F.; CARVALHO, A. P. M.; DAVI, R. F. L.. Perfil epidemiológico da hanseníase na microrregião de Araçuaí e sua relação com ações de controle. Escola Anna Nery Revista de Enfermagem, v.15, n.1, p.62-67, 2011.

LIMA, M. M.; MOREIRA, A. M.. Perfil Epidemiológico da Hanseníase em um município de Minas Gerais: uma análise retrospectiva. Revista Prevenção de Infecção e Saúde, v.1, n.3, p.1-10, 2015.

MELÃO, S.; BLANCO, L. F. O.; MOUNZER, N.. Perfil epidemiológico dos pacientes com hanseníase no extremo sul de Santa Catarina, no período de 2001 a 2007. Revista da Sociedade Brasileira de Medicina Tropical, v.44, n.1, p.7984, 2011.

MIRANZI, S. S. C.; PEREIRA, L. H. M.; NUNES, A. A.. Perfil epidemiológico da hanseníase em um município brasileiro, no período de 2000 a 2006. Revista da Sociedade Brasileira de Medicina Tropical, v.43, n.1, p.62-67, 2010.

MONTEIRO, L. D.; MELO, F. R. M.; BRITO, A. L.; LIMA, M. S.; ALENCAR, C. H.; JORGE, H.. Tendências da hanseníase no Tocantins: um estado hiperendêmico do Norte do Brasil, 2001-2012. Cadernos de Saúde Pública, v.31, n.5, p.971-980 2015. DOI: https://doi.org/10.1590/0102-311X00075314

OLIVEIRA, K. S.; SOUZA, J.; BEZERRA, R.; ZILLY, A.; SILVASOBRINHO, R. A.. Avaliação dos indicadores epidemiológicos e operacionais para a hanseníase em municípios prioritários no estado do Paraná, 2001 a 2010. Epidemiologia e Serviços de Saúde, v.24, n.3, p.507-516, 2015.

RODRIGUES, V. V.. Perfil epidemiológico dos casos novos de hanseníase no município de Teófilo Otoni/MG: uma análise de 2001 a 2008. Monografia (Especialização em Atenção Básica em Saúde da Família) - Universidade Federal de Minas Gerais, Teófilo Otoni, 2010.

SANTOS, A. S.; CASTRO, D. S.; FALQUETO, A.. Fatores de risco para transmissão da Hanseníase. Revista Brasileira de Enfermagem, v.61, p.738-743, 2008.

TALHARI, S.; PENNA, G. O.; GONÇALVES, H. S.; OLIVEIRA, M. L.. Hanseníase. 5 ed. Manaus: Dilivros, 2014.

VERONESI, R.; FOCACCIA, R.. Tratado de infectologia. 2 ed. São Paulo: Atheneu, 2002. 